\title{
Echocardiographic evaluation of left ventricular strain in severe aortic stenosis with therapeutic implications and risk stratification
}

\author{
Anna Teresa Goździk ${ }^{1, A-D}$, Marek Jasiński, ${ }^{1, C, E, F}$, Waldemar Goździk ${ }^{2, C, E, F}$ \\ ${ }^{1}$ Department and Clinic of Cardiac Surgery, Wroclaw Medical University, Poland \\ ${ }^{2}$ Department and Clinic of Anesthesiology and Intensive Therapy, Wroclaw Medical University, Poland
}

A - research concept and design; $B$ - collection and/or assembly of data; $C$ - data analysis and interpretation;

$D$ - writing the article; $E$ - critical revision of the article; $F$ - final approval of the article

Address for correspondence

Anna Goździk

E-mail:anna.gozdzik@umed.wroc.pl

Funding sources

None declared

Conflict of interest

None declared

Received on March 29, 2018

Reviewed on 0ctober 6, 2018

Accepted on February 18, 2019

Published online on August 14, 2019

\begin{abstract}
Degenerative aortic stenosis (AS) is an increasingly common acquired valvular heart disease in adults due to the extension of life expectancy in the population of developing countries. The occurrence of calcifications and associated severe aortic stenosis (SAS) increases with age and affects approx. 3-5\% of people over 75 years of age. The basis for the decision on the date and type of therapy is echocardiographic evaluation of the severity of the AS and left ventricular (LV) function as well as clinical signs. It appears that the use of newer, more precise methods in echocardiography, especially in patients with preserved ejection fraction (pEF), may change our management in qualifying for valve replacement, especially in asymptomatic patients with SAS. The aim of this review study is echocardiographic strain analysis and evaluation of strain of LV myocardial fibers in patients with SAS, using the speckle tracking echocardiography (STE). This evaluation allows for risk stratification of a valve disease and the choice of the appropriate therapy method.
\end{abstract}

Key words: aortic stenosis, speckle tracking echocardiography, strain
Cite as

Goździk AT, Jasiński M, Goździk W. Echocardiographic evaluation of left ventricular strain in severe aortic stenosis with therapeutic implications and risk stratification. Adv Clin Exp Med. 2019;28(9):1271-1279. doi:10.17219/acem/104553

DOI

10.17219/acem/104553

Copyright

Copyright by Author(s)

This is an article distributed under the terms of the

Creative Commons Attribution Non-Commercial License

(http://creativecommons.org/licenses/by-nc-nd/4.0/) 


\section{Introduction}

Degenerative aortic stenosis (AS) is an increasingly common acquired valvular heart disease in adults due to the extension of life expectancy in the population of developing countries. The occurrence of calcifications and associated severe aortic stenosis (SAS) increases with age and affects approx. $3-5 \%$ of people over 75 years of age. ${ }^{1}$ The process of forming aortic valve lesions results from the deposition of lipids, infiltration of macrophages and $\mathrm{T}$ lymphocytes in the endothelium of aortic cusps and stimulation of their calcification processes (Fig. 1).

The consequence of an increase in AS is pressure overload and the resulting concentric left ventricular hypertrophy $(\mathrm{LVH})$. At this stage, the cardiac output and filling pressure are normal. The left ventricular (LV) remodeling, in addition to stenosis, is also influenced by other factors such as the age and sex of the patient, e.g., in women the process of faster calcification of the valve and quicker appearance of the disease symptoms are observed. ${ }^{2}$ The other important factors are the following: genetic diversity in the renin-angiotensin system, comorbid coronary artery disease, arterial hypertension, and significant aortic regurgitation. In patients with associated arterial hypertension, the LV afterload increases, which at a certain stage of the disease causes a decrease in the stroke volume and progressive deterioration of myocardial function. In patients with SAS and associated hypertension, a reduced survival rate was observed. ${ }^{3}$

A careful diagnosis of AS is important, not only regarding the size of the stenosis and transvalvular gradient of stenosis, but also the function of the LV and its impact on pulmonary pressure, the right ventricle and the left atrium. The current criteria for echocardiographic diagnosis of SAS are presented in the ESC/EACTS recommendations of 2017. ${ }^{4}$

Surgical treatment is level B class I recommendation in symptomatic patients with severe AS confirmed with echocardiography. Level C class I recommendation includes asymptomatic patients with SAS and with reduced

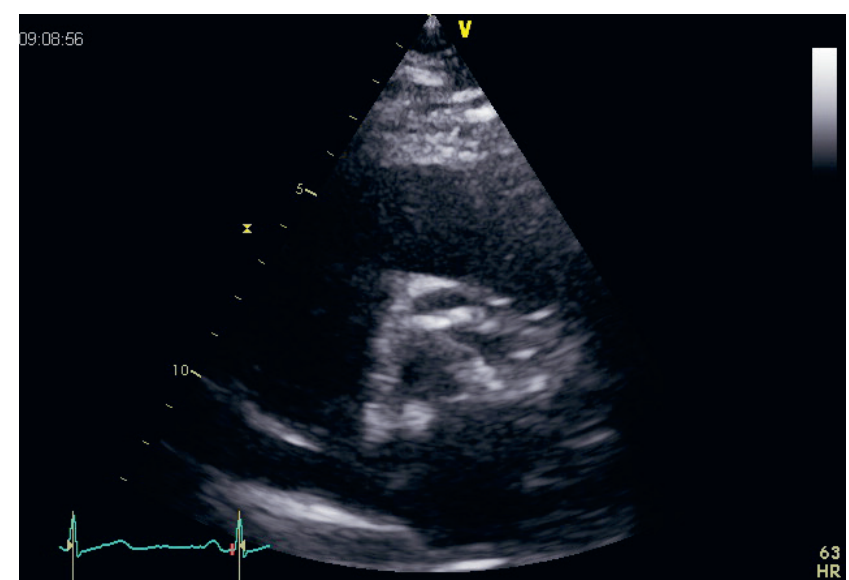

Fig. 1. Image of SAS in short axis parasternal transthoracic view left ventricular ejection fraction (LVEF) $<50 \%$, as well as asymptomatic patients with SAS and abnormal exercise test results in which symptoms were manifested during exercise clearly resulting from AS. Surgical aortic valve replacement (AVR) is recommended for patients from the low surgical risk group (STS or EuroSCORE II $<4 \%$ or logistic EuroSCORE II $<10 \%$ without other risk factors not included in the abovementioned scales such as frailty syndrome, porcelain aorta or history of chest irradiation) - this is level B class I recommendation. Transcatheter aortic valve implantation (TAVI) is recommended for patients who, in the opinion of the Heart Team, are not suitable candidates for AVR - level B class I recommendation. ${ }^{5}$

Management of patients in the asymptomatic period with preserved ejection fraction (pEF) remains debatable. The onset of symptoms and the deterioration of the LV function are mostly associated with poor prognosis. Left ventricular ejection fraction is still the basic echocardiographic parameter for assessing myocardial function. It appears that the use of newer, more precise methods in echocardiography, especially in patients with $\mathrm{pEF}$, may change our management policy in the field of qualifications for AVR, especially in asymptomatic patients with SAS.

The aim of this review is an echocardiographic analysis and evaluation of a strain of LV myocardial fibers in patients with SAS using the speckle tracking echocardiography (STE).

\section{Risk factors for aortic stenosis}

The process of AS formation is the result of many factors including clinical, genetic and anatomical factors. Clinical factors include old age, male sex, elevated low-density lipoprotein (LDL) and lipoprotein(a) (Lp(a)), arterial hypertension, smoking, diabetes, and metabolic syndrome. An increased risk of AS is also present in patients after mediastinal irradiation. ${ }^{6}$

The presence of bicuspid aortic valve as a congenital defect is more conducive to degenerative valve lesions. The congenital polymorphism of $\mathrm{Lp}(\mathrm{a})$ and an elevated level of this lipoprotein in serum are associated with the possibility of inheriting the tendency for calcification to form on the valves. The increase in AS from the period of sclerosis in which cusp calcifications and thickening develop to hemodynamically significant stenosis takes on average from 2 to 5 years in $10-15 \%$ of patients. ${ }^{7}$

In the period when the AS is mild and with echocardiographic evaluation of the transvalvular velocity results of about $2 \mathrm{~m} / \mathrm{s}$ are noted, progression occurs in almost all patients and most of them require AVR. The average increase in AS in adults from a mild to moderate defect is manifested by a change in the following parameters: an increase in the transvalvular velocity by $0.3 \mathrm{~m} / \mathrm{s} /$ year, an increase in the mean transvalvular gradient by $7 \mathrm{~mm} \mathrm{Hg} /$ year and a decrease in the effective orifice area (EOA) by $0.1 \mathrm{~cm}^{2} /$ year. $^{8}$ 
When significant valve stenosis is found, it is very important to differentiate between symptomatic and asymptomatic patients. A patient with the typical symptoms of SAS (angina pectoris, syncope, arrhythmias, and heart failure) requires intervention in the form of AVR within 30 days of symptoms onset, as this is the only way to reduce the symptoms and decrease mortality in this group of patients. The mortality of these patients over a 2-year period without surgical intervention is $50 \%{ }^{9}$

The management of asymptomatic patients with significant valve stenosis is still under debate. Some authors suggest earlier AVR based on 2 factors: 1. The risk of sudden cardiac death (about 1\% per year in these patients), which approaches the risk of a surgical intervention averaging to about $2.5 \%$ and even less in experienced cardiac surgery centers. 2 . The risk of complications, which may be even higher in patients with severe aortic valve calcification and a rapid increase of peak transvalvular velocity, e.g., $>5.5 \mathrm{~m} / \mathrm{s}$, abnormal reaction or symptoms induced by the exercise test or a high level of serum natriuretic peptides. ${ }^{10}$

In observational studies covering the issues of natural history of asymptomatic patients with SAS, it was found that about $1 / 3$ of them become symptomatic within 2 years and $2 / 3$ of them underwent AVR or sudden cardiac death within 5 years. ${ }^{11}$

Survival in asymptomatic, non-operated patients is reported to be $99 \%, 98 \%$ and $93 \%$, respectively, at the end of 1,2 and 5 years of follow-up. It is worth noting that the survival rate of these patients was similar to the healthy population at a similar age and of the same sex..$^{12}$ The longterm prognosis worsens with the onset of symptoms.

In the management of an asymptomatic patient, the following principles should be respected: identification of high-risk patients, education for the symptoms of AS, periodic follow-up examinations, treatment of additional diseases, and optimization of the AVR time.

\section{Left ventricular pathophysiology in aortic stenosis}

In order to optimize the time for AVR in SAS, it is necessary to evaluate in detail the hemodynamic consequences of the confirmed disease. The LV pressure overload in SAS results in a change in its geometry and function (hypertrophy, dilation and a decrease in EF). In order to maintain the heart function ( $\mathrm{pEF}$ ), the high stress of the ventricular wall leads to the hypertrophy of the middle layer of the muscle. Further increase in stress in this layer gradually intensifies myocyte hypertrophy, with time leading to fibrosis of extracellular space. At this stage, a progressive deterioration in heart function is noted, although the stroke volume and ejection fraction (EF) may remain normal. Development of valve stenosis causes progressing changes in myocardium, along with the death of cardiomyocytes and focal replacement fibrosis. Apart from the degree of the valve stenosis, muscle hypertrophy is also affected by the following factors: sex, age, genetically conditioned angiotensin-aldosterone system disorders, concomitant coronary artery disease, arterial hypertension, or significant aortic regurgitation.

In patients with concomitant arterial hypertension, there is an increase in peripheral vascular resistance which, accompanied by AS, increases the stress of the ventricular wall, at a later stage causing an increase in the afterload. The consequence of this may be a decrease in cardiac output, damage to the function of the myocardium and reduced survival rate. Long-term significant valve stenosis causes the exhaustion of cardiac compensatory mechanisms and contributes to an increase in LV diastolic stiffness and impairment of its filling function. The LV becomes incapable of maintaining an adequate stroke volume and this condition is called "afterload mismatch". During this period, there is no significant damage to the LV systolic function and EF is preserved ( $\mathrm{pEF}$ ), but only the correction of the heart defect results in a complete, beneficial improvement in LV function. However, diastolic dysfunction and unfavorable response to physical exercise in patients with pEF may still continue for many years after AVR. ${ }^{13}$ A compensatory change of the LV geometry is associated with an increase in the relative wall thickness (RWT) with the unchanged function of the radial and circumferential fibers in the middle layer of the LV wall, which in turn allows the normal EF (pEF) to be maintained.

A common phenomenon in AS is impaired coronary flow reserve with decreased flow in the subendocardial layer, even in the absence of lesions in the coronary vessels. This is due to the severity of the valve stenosis and the associated LV hemodynamic load and the shortened diastolic perfusion time. These phenomena may be the basis for fiber dysfunction in the longitudinal dimension.

Early identification of myocardial contractility damage symptoms is important, especially in asymptomatic patients with AS, and helps in their earlier qualification for AVR surgery. Based on data from cardiovascular magnetic resonance, it has been found that LV remodeling in SAS in the first period takes the form of extracellular matrix diffuse fibrosis and is reversible, similarly to myocardial cellular hypertrophy. However, cardiomyocytes necrosis with replacement fibrosis (scar) is an irreversible process.

\section{Echocardiography}

Echocardiography plays a central role in the assessment and management of patients with SAS. This examination method helps to stratify the risk in a heterogeneous group of asymptomatic patients with SAS. The main echocardiographic technique used to assess the severity of AS is Doppler echocardiography. The use of Doppler echocardiography, due to its high availability and versatility, 
received Class I recommendations. Cardiac catheterization is not routinely performed. The use of this method is reserved for patients with ambiguous results of non-invasive examinations. The criterion for the diagnosis of SAS according to current recommendations (ESC and EACTS) of 2017 is as follows:

1. Peak transvalvular velocity $>4 \mathrm{~m} / \mathrm{s}$;

2. Mean gradient $(\mathrm{MG})>40 \mathrm{~mm} \mathrm{Hg}$;

3. Effective orifice area (EOA) $<1.0 \mathrm{~cm}^{2}$;

4. EOAI $<0.6 \mathrm{~cm}^{2} / \mathrm{m}^{2}$.

Unfortunately, results that do not comply with the guidelines may appear in clinical practice. This occurs in approx. $25-30 \%$ of patients with SAS and preserved left ventricular ejection fraction (pLVEF). In this situation, there is a problem with the assessment of the severity of stenosis and the conditions where, e.g., EOA $<1.0 \mathrm{~cm}^{2}$ and $\mathrm{MG}<40 \mathrm{~mm} \mathrm{Hg}$ are inconsistent with the guidelines for diagnosing SAS, making the decision to qualify the patient for AVR surgery difficult. It has been repeatedly stated that the EOA parameter obtained from the calculation of the continuity equation can be miscalculated (a more accurate measurement is obtained in 3D or computed tomography (CT)) and, therefore, it was assumed that the evaluation of the severity of AS is based mainly on the MG. The quite common underestimation of the severity of AS is associated with late qualification of patients for AVR and with poor long-term prognosis. ${ }^{14}$

Besides the interpretation of echocardiographic parameters, it is important to assess the blood pressure and the functional status of a patient. Patients with high blood pressure should be reevaluated after its normalization. ${ }^{15}$

Taking into account the assessed parameters, 4 categories of AS can be defined:

1. High-gradient AS, EOA $<1.0 \mathrm{~cm}^{2}, \mathrm{MG}>40 \mathrm{~mm} \mathrm{Hg}$ - the stenosis can be determined as severe regardless of whether LVEF and flow are normal or decreased.

2. Low-flow, low-gradient AS with decreased EF - EOA $<1.0 \mathrm{~cm}^{2}, \mathrm{MG}<40 \mathrm{~mm} \mathrm{Hg}, \mathrm{EF}<50 \%$, indexed stroke volume (SVI) $\leq 35 \mathrm{~mL} / \mathrm{m}^{2}$.

In this case, a dobutamine test with a low dose of dobutamine is recommended in order to differentiate a true-severe from pseudo-severe AS, which was defined as increasing the EOA $>1.0 \mathrm{~cm}^{2}$ along with normalization of flow. The presence of flow reserve (contractility reserve, increasing the stroke volume $>20 \%$ ) is of prognostic importance, because it is associated with a better long-term prognosis. ${ }^{16}$

3. Low-flow, low-gradient AS with pEF called paradoxical low-flow - EOA $<1 \mathrm{~cm}^{2}, \mathrm{MG}<40 \mathrm{~mm} \mathrm{Hg}, \mathrm{EF} \geq 50 \%$, $\mathrm{SVI} \leq 35 \mathrm{~mL} / \mathrm{m}^{2}$.

This occurs typically in elderly patients, it is associated with small size of the LV and marked LVH and often with a history of arterial hypertension. ${ }^{17}$

The diagnosis of SAS in this case requires ruling out measurement errors and other reasons for such an echocardiographic image. The degree of calcification assessed by means of multi-slice computed tomography (MSCT) is associated with the severity of stenosis and with prognosis. ${ }^{18}$ Therefore, the evaluation of this parameter is of increasing importance in such cases.

4. Normal-flow, low-gradient AS with $\mathrm{pEF}, \mathrm{EOA}<1 \mathrm{~cm}^{2}$, $\mathrm{MG}<40 \mathrm{~mm} \mathrm{Hg}, \mathrm{EF} \geq 50 \%$, and SVI > $35 \mathrm{~mL} / \mathrm{m}^{2}$. Such patients generally present with moderate grade AS.

The second step in the echocardiographic assessment of AS is the assessment of its impact on the geometry and function of the LV and other heart structures. Linear LV dimensions measurements should be performed in all patients based on recommendations for further estimation of LV mass and RWT in order to classify the type of LV remodeling (Fig. 2).

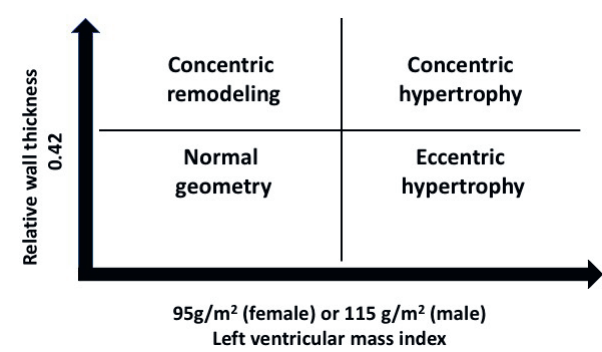

Fig. 2. Left ventricular measurements of left ventricular mass index and RTW

The estimation of the LV systolic function can be obtained with measuring the endocardial movement during the LV systole and diastole. Parameters - shortening fraction (SF) and EF - are calculated from the obtained parameters. Unfortunately, in the presence of concentric muscle hypertrophy ( $\mathrm{LVH}$ ) which we find in AS, there is often an overestimation of the systolic function. Another important parameter is mitral annular plane systolic excursion (MAPSE), which reflects the global LV longitudinal systolic function and is a sensitive index of systolic dysfunction comparable to LVEF. The MAPSE value decreases with the increase in stenosis severity, regardless of $\mathrm{LVH}$, indicating a direct association with the increase in hemodynamic load in AS. A decrease in MAPSE is associated with an increase in subendocardial fibrosis. The cut-off point for the MAPSE value is $9 \mathrm{~mm}$ and is of high value in the differentiation between SAS and moderate AS. ${ }^{19}$ The exact calculation of LV stroke volume (using LV outflow tract velocity time integral and diameter) must be included in the echocardiographic evaluation of AS patients, especially in patients with SAS (AV calculation $<1.0 \mathrm{~cm}^{2}$ ), pEF $(>50 \%)$ and a low mean transvalvular gradient ( $\mathrm{MG}<40 \mathrm{~mm} \mathrm{Hg}$ ). A cut-off points value $<35 \mathrm{~mL} / \mathrm{m}^{2}$ is an essential criterion for the diagnosis of paradoxical low-flow $\mathrm{AS}^{20}$

Left ventricular ejection fraction below $50 \%$ is the only parameter of systolic LV function which obliges us to intervene in patients with SAS. But it is not a good parameter of myocardial contractility, because it is mainly determined by the radial function which can be normal for a long time, even in the presence of subendocardial fibrosis. At this point, we should remember about the structure 
of the LV muscle. It has a double helical structure. Subepicardial fibers are going circumferentially, in the mid-wall part in radial and subendocardial in longitudinal direction. The longitudinal and circumferential fibers shorten, and the radial fibers thicken during systole.

Nowadays, it is recognized that LVEF is not a sensitive marker of myocardial dysfunction, and an impairment in EF is often a late manifestation that may not be reversible. ${ }^{21}$ In symptomatic SAS, AVR is beneficial for improving their cardiac function. However, some patients do not experience cardiac function improvement after a successful AVR, and some of them even experience deterioration. Therefore, there is a great need to introduce a method that specifically evaluates the complex function of the heart muscle, which will allow patients to be qualified earlier for AVR surgery and will improve long-term prognosis. This role is attributed to tissue Doppler imaging (TDI) and two-dimensional STE (2D-STE). The introduction of 3D echo imaging increases the diagnostic possibilities to an even greater extent.

\section{Tissue Doppler imaging}

Tissue Doppler imaging (TDI) is used to assess global and segmental LV systolic function. The determination of tissue movement in TDI is based on the same principles as the calculation of the flowing blood velocity in pulse wave Doppler or color Doppler. The use of TDI supported by numerous clinical trials allowed detection of changes in LV function in patients with SAS and pLVEF. Peak annular systolic velocities $\left(S^{\prime}\right)$ were significantly reduced in patients with SAS and pLVEF. ${ }^{22}$ Reductions of early diastolic mitral annular velocity (e') and higher values of E/E' ratio were found in asymptomatic patients with moderate AS, indicating an early impairment of diastolic function. ${ }^{23}$ The prognostic value of TDI (S', E' and A' velocities) was evaluated in 183 asymptomatic patients with SAS and pLVEF. Patients who developed the symptoms had lower $S$ 'values, suggesting subclinical LV dysfunction compared to asymptomatic patients $(6.6 \mathrm{~cm} / \mathrm{s}$ vs $7.2 \mathrm{~cm} / \mathrm{s}, \mathrm{p}=0.03) .{ }^{24}$

Novel myocardial deformation imaging (strain and strain rate and torsion) using STE has been proposed as a sensitive approach of assessing intrinsic myocardial contractility.

\section{D speckle tracking echocardiography}

The 2D-STE technique is a relatively new method for assessing myocardial function. It allows the assessment of the global and segmental function of the heart in an objective, quantitative manner, independent from the angle of analysis. Interactions between the ultrasound beam and muscle fibers smaller than the ultrasonic wavelength are the reason for the heterogeneity of the grayscale echocardiographic image. The resulting point grains (speckles) are specific acoustic markers. The STE is based on the analysis of the change in their position in two-dimensional highresolution echocardiographic images in the cardiac cycle. By tracking the displacement of acoustic markers using modern algorithms, one can obtain data on the velocity and the value of displacement, the strain rate and the strain of myocardium in 3 special directions: longitudinal, radial and circumferential, as well as on the LV rotation. ${ }^{25}$

The parameters assessed using STE include:

1. Strain - a measurement determining the degree of deformation of the analyzed myocardium area in relation to its initial position. It is expressed as a percentage. It is assumed to take negative values when the strain is shortening or thinning and positive when the strain consists in lengthening or thickening.

Types of strains:

A. Longitudinal strain (LS) - myocardial deformation directed from the base to the apex of the heart, determined in apical 3, 4.2-chamber views. In a healthy heart, in systole the longitudinal dimension of the LV is shortened, hence the negative values of longitudinal strain. Longitudinal strain allows data to be obtained on segmental and global LV function (global longitudinal strain - GLPS). Topographic presentation of peak longitudinal strain values is possible in the form of a planar map for 17 segments of the LV (bull eye) (Fig. 3).

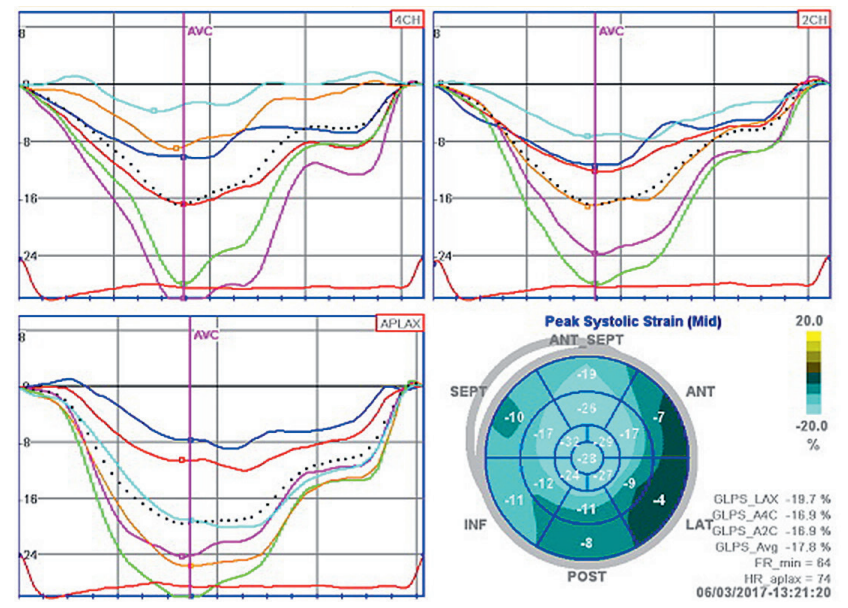

Fig. 3. Left ventricular longitudinal strain measured using STE in SAS

B. Radial strain (RS) - myocardial deformation directed to the center of the LV cavity evaluated in parasternal short axis views. In systole, the myocardium thickens; hence, the radial strain takes positive values in this phase of the cycle.

C. Circumferential strain (CS) - myocardial deformation consisting in the shortening of muscle fibers in a circular dimension leading to a reduction of the LV circumference. It is estimated in parasternal short axis views. It takes negative values. 
2. Strain rate $1 / \mathrm{s}(\mathrm{SR})$ - a parameter showing the rate of the myocardium strain. It is calculated from the quotient of the velocity difference of 2 points in the area under examination and the distance between these points.

Using the algorithms based on acoustic marker tracking, it is also possible to analyze the LV rotatory mechanics. The LV rotatory strain is shown by:

1. Rotation $(\mathrm{R})$ is a parameter describing the LV rotatory movement in the short-axis cross-section around a point located in the center of the mass of the LV. It assumes the value of the angle by which the myocardium deviates in the cross-section of interest and in a specific phase of the cardiac cycle in relation to its initial value. The rotational movement of the apex and base of the heart takes place in opposite directions. The apex rotates counterclockwise and the angle of rotation takes positive values. The base of the heart rotates in the clockwise direction and takes negative values.

2. Left ventricular systolic twist (Twist - in degrees) - the absolute difference between the maximal value of rotation at the level of the apex (Ar) and the maximal value of rotation at the level of the heart base (Br) (Fig. 4).

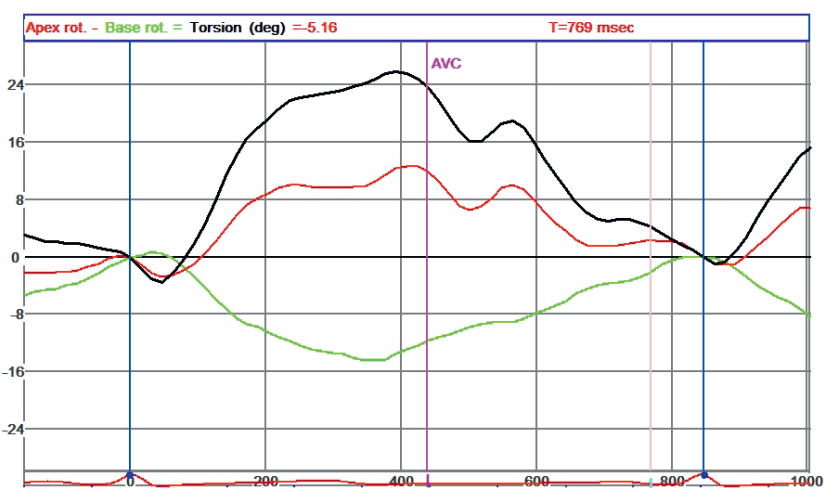

Fig. 4. Rotation and twist measured using STE in SAS

3. Systolic twist standardized by the LV longitudinal dimension (Torsion - in \% cm) - the quotient of LV systolic twisting and the mean value of LV longitudinal dimension of the LV in diastole - the distance between the apex and the base of the heart in 4 and 2 chamber views.

4. Diastolic LV torsional deformation (Untwist - in degrees) - the absolute difference between the values of LV torsion at the apex and at the base of the heart in diastole. In the early phase of diastole, the potential energy accumulated in the heart interstitium at the active stage of contraction is released and the myocardium passively deforms at individual levels in directions opposite to those observed in the phase of the systole.

5. Post-systolic shortening (PSS) is the difference between the maximum value of shortening during the entire cardiac cycle and the shortening values at the aortic valve close (AVC) point. It is presented in the form of the postsystolic index (PSI), which is the quotient of the PSS and the maximum value of shortening during the cardiac cycle, expressed as a percentage.

The results from clinical studies using 2D-STE in patients with SAS and pLVEF confirmed a significant decrease in LV longitudinal strain mainly in basal segments and showed its impact on exercise capacity and pure prognosis in asymptomatic patients. In these patients, more cardiac events and abnormal exercise responses were observed with a sensitivity of $68 \%$ and a specificity of $77 \% .{ }^{26}$ The measurements of longitudinal strain are not only related to the severity of AS but also to the type of LV remodeling, with lower values in patients with higher LV mass and RWT. ${ }^{27} \mathrm{Ng}$ et al. have showed that along the progression of AS severity there is a progressive impairment of strain in all kinds of muscle fibers $(\mathrm{p}<0.001){ }^{28} \mathrm{De}-$ creased global longitudinal strain in AS groups was also reported by Miyazaki et al. ${ }^{29}$ and Delgado et al. ${ }^{30}$ Galli et al. were studying the influence of increasing valvular-arterial impedance (ZVa) on the longitudinal, circumferential and radial components of $L V$ mechanics in patients with AS. The ZVa is a recently introduced parameter which permits the evaluation of global LV afterload in AS. It was calculated as the sum of the systolic arterial pressure and the mean transvalvular pressure gradient divided by SVI. They showed that global longitudinal strain (GLS) and global circumferential strain (GCS) are often reduced in the presence of increased ZVa. Global radial strain (GRS) was significantly altered only in patients with the highest ZVa. This means that mid-wall radial fibers are far less sensitive to LV afterload with respect to subendocardial longitudinal fibers. The radial fibers compensate for the reduction in longitudinal and CS, which contributes to maintaining the global LV function. When the global LV dysfunction is set, GRS significantly decreases and becomes strongly influenced by LV afterload. ${ }^{31}$ Donal et al. ${ }^{32}$ made the same observations in an animal model. The chronic exposure to a high global LV load developed the impairment of myocardial contractile function. Marechaux et al. ${ }^{33}$ have shown a negative correlation between ZVa and GLS ( $r=-0.41$, $\mathrm{p}<0.0001)$.

The value of LV twist depends to a large extent on preload (it increases with preload increase) and afterload (it decreases with afterload increase as in AS). ${ }^{34}$ Disturbed values of the torsion rate have been shown in clinical trials in patients with LV diastolic dysfunction secondary to AS. ${ }^{35}$ The PPS parameter has also been proven to be a sensitive but nonspecific index of LV muscle dysfunction. ${ }^{36}$ Stress testing (the low dobutamine or exercise stress tests) is recommended for asymptomatic patients with SAS, especially for the differentiation of true-severe from pseudo-severe AS. Global 2D LS has the big possibility to provide more accurate information about LV function during stress testing. ${ }^{37}$ Donal et al. ${ }^{38}$ and Lafitte et al. ${ }^{26}$ have both showed that lower LS was detected in AS patients with the positive exercise test than in AS patients with the negative test. The assessment of LV contractile reserve 
using the low dobutamine stress test has clear prognostic implication in patients with low-flow low-gradient AS and reduced LVEF. A decrease or limited increase in LVEF during exercise is associated with a markedly reduced midterm cardiac event-free survival. ${ }^{39}$ Global longitudinal strain during exercise allows more precise detection of latent LV systolic dysfunction than LVEF.

The elevated LV afterload can induce alterations in torsion. Patients with AS manifested increased apical rotation $\left(13^{\circ} \pm 5.8^{\circ}\right.$ vs $\left.7.7^{\circ} \pm 2.6^{\circ}, \mathrm{p}<0.001\right)$ and twist $\left(19.7^{\circ} \pm 5.7^{\circ}\right.$ vs $\left.12.9^{\circ} \pm 3.2^{\circ}, \mathrm{p}<0.001\right)$, compared to a healthy group, while basal rotation remained normal. ${ }^{40}$ Popescu et al. and van Dalen et al. ${ }^{41}$ have had similar observations.

Carasso et al. ${ }^{42}$ have demonstrated the differential mechanics between compensation and decompensation of AS using strain imaging. Compensatory LV showed an increase in the apical rotation angle in patients with $>50 \%$ LVEF and high CS in patients with pLVEF, whereas decompensation showed a decrease in CS and the rotation angle.

\section{Prognostic value of echocardiographic parameters in aortic stenosis}

The management of patients with asymptomatic SAS was also observed by Lech et al. They have found an increase in GLS during the exercise test in patients with SAS, but smaller than in the control group. The observation group was small (50 points) and further studies are needed. The authors have indicated a significant role of the relationship between the LV mass and GLS. A degree of myocardial hypertrophy could be a stronger argument for qualifying asymptomatic patients for surgical treatment. This requires further research. ${ }^{43}$ In their latest research, $\mathrm{Ng}$ et $\mathrm{al} .{ }^{44}$ have presented the largest study to date, which included 688 AS patients, of which 294 had SAS. They observed patients with a wide range of LVEF and AS severity. The conclusions are the following: the LV GLS is an independent and superior prognosticator compared with LVEF in patients with AS. Severe AS patients with normal LVEF may present evidence of myocardial dysfunction and have an increased mortality risk, similar to that of SAS patients with impaired LVEF (log-rank $\mathrm{p}=0.34)$. Therefore, LV GLS can further stratify risk in SAS patients and may influence the optimal timing of AVR. The prognostic value of LV GLS was confirmed by Kusunose et al. in a big group of AS patients $(n=395)$, mean age $70 \pm 14$ years, $57 \%$ men, EOA $<1.3 \mathrm{~cm}^{2}$ and pLVEF. The cut-off value of LV GLS > $-12.1 \%$ was independently connected with increased mortality. ${ }^{45}$ The patients with inconsistent grades of AS are very often asymptomatic. Lancellotti et al. have proposed a risk stratification model founded on the characteristic of flow and gradient. The patients with low-flow, lowgradient SAS have the lowest cardiac event-free survival compared with patients with low-flow, high-gradient SAS. The patients with normal-flow, high-gradient and normalflow, low-gradient SAS demonstrate a higher frequency of survival. ${ }^{46}$

Myocardial deformation improved progressively after a successful AVR ${ }^{47}$ In a study by Rosa et al. it was indicated that there was no significant improvement in the strain observed 1 week after AVR along with unchanged LVEF; a longer follow-up still showed increased deformation 6 months after AVR. ${ }^{48}$ Carasso et al. have showed that longitudinal systolic strain increased from $-12.8 \% \pm 1.7 \%$ to $-15.9 \% \pm 2.2 \%$, whereas mid-LV circumferential strain decreased from $-27.0 \% \pm 5.1 \%$ to $-22.3 \% \pm 4.9 \%$ at an early follow-up post AVR ( $7 \pm 3$ days) in AS patients with pLVEF. In another study, Carasso et al. have found in research on AS patients with $\mathrm{EF}<50 \%$ that the circumferential strain that was decreased before AVR had a significant improvement after AVR. ${ }^{49} \mathrm{~A}$ twist and apical rotation increased in compensated patients and normalized after AVR. Normalization of twist in decompensated patients after AVR was uncertain.

In echocardiographic imaging, we can also use the 3D STE test, which allows simultaneous imaging of strain in 3 perpendicular directions, thus giving us the unique possibility of performing a simultaneous quantitative analysis of strain in the longitudinal and circumferential directions and obtaining the so-called area strain. The advantage of 3D STE over 2D STE is the ability to obtain LV strain values based on data from 1 cardiac cycle. The main limitation of 3D STE, however, is the insufficient quality of echocardiographic images, from which 3D reconstructions are created. Others include a worse volume rate than in the case of 2D STE, and a lower level of validation and of clinical usefulness assessment. ${ }^{50}$

\section{Conclusions and perspectives}

The consequences of increased afterload on LV should always be taken into account for the comprehensive assessment of patients with AS. Besides the conventional assessment of LV mass and EF, the measurements of LV strain (GLS) and myocardial fibrosis (when needed estimated by CMR) will probably be increasingly used in the decision-making process in patients with AS.

Echocardiographic parameters of longitudinal function are strongly linked to the extent of myocardial fibrosis, which has clear prognostic implications. Mid-wall myocardial fibrosis was associated with an 8-fold increase in allcauses mortality in patients with significant AS, while focal fibrosis was an independent predictor of increased preoperative risk and mortality in patients with AS undergoing surgical AVR. ${ }^{51,52}$

Therefore, the echocardiographic assessment of LV LS allows for the estimation of LV subendocardial fibrotic changes and becomes a tool for risk stratification in patients 
with SAS. Nowadays the clinical utility of GLS is hindered by the lack of standardization on different echo cardiographic machines and the lack of specific cut-off values.

Exercise echocardiography with the application of GLS may provide the incremental prognostic value by assessing both exercise-induced symptoms and changes in valve hemodynamics, LV function and pulmonary pressure. This approach can aid in timing the intervention in asymptomatic patients with SAS and stratify risk in patients undergoing AVR.

\section{References}

1. Carabello BA. Introduction to aortic stenosis. Circ Res. 2013;113(2): 179-185.

2. Aggarwal S, Clavel MA, Messika-Zeitoun D, et al. Sex differences in aortic valve calcification measured by multidetector computed tomography in aortic stenosis. Circ Cardiovasc Imaging. 2013;6(1): 40-47.

3. Kadem L, Dumessnil JG, Rieu R, Durand LG, Garcia D, Pibarot P. Impact of systemic hypertension on the assessment of aortic stenosis. Heart. 2005;91(3):354-361.

4. Baumgartner H, Falk V, Bax J, et al; ESC Scientific Document Group. ESC/EACTS Guidelines for the management of valvular heart disease. Eur Heart J. 2017;36(38):2739-2791.

5. Thourani VH, Suri RM, Gunter RL, et al. Contemporary real-world outcomes of surgical valve replacement in 141,905 low-risk, intermediate-risk, and high-risk patients. Ann Thorac Surg. 2015;99(1):55-61.

6. Kurtz CE, Otto CM. Aortic stenosis: Clinical aspects of diagnosis and management with 10 illustrative case reports a 25 -year experience. Medicine (Baltimore). 2010;89(6):349-379.

7. Eveborn GW, Schrimer H, Heggelund G, Lunde P, Rasmussen K. The evolving epidemiology of valvular aortic stenosis: The Troms $\varnothing$ study. Heart. 2013;99(6):396-400.

8. Otto CM, Prendergast B. Aortic valve stenosis: From patients at risk to severe valve obstruction. NEngl J Med. 2014;371(8):744-756.

9. Joint Task Force on Management of valvular heart disease of European Society of Cardiology (ESC); European Association for CardioThoracic Surgery (EACTS); Vahanian A, Alfieri O, Andreotti F, et al. Guidelines on the management of valvular heart disease (version 2012). Eur Heart J. 2012;33(19):2451-2496.

10. Rosenhek R, Binder T, Porenta G, et al. Predictors of outcome in severe, asymptomatic aortic stenosis. N Eng/ J Med. 2000;343(9):611-617.

11. Rosenhek R, Zilberszac R, Schemper M, et al. Natural history of very severe aortic stenosis. Circulation. 2010;121(1):151-156.

12. Otto CM, Burwash IG, Legget ME, et al. Prospective study of asymptomatic valvular aortic stenosis. Clinical, echocardiographic and exercise predictors of outcome. Circulation. 1997;95(9):2262-2270.

13. Ross J Jr. Afterload mismatch in aortic and mitral valve disease: Implications for surgical therapy. J Am Coll Cardiol. 1985;5(4):811-826.

14. Minners J, Allgeier M, Gohlke-Baerwolf C, et al. Inconsistencies of echocardiographic criteria for the grading of aortic valve stenosis. Eur Heart J. 2008;29(8):1043-1048.

15. Baumgartner $\mathrm{H}$, Hung J, Bermejo J, et al. Focus update on the echocardiographic assessment of aortic valve stenosis: EAE/ASE recommendations for clinical practice. Eur Heart J Cardiovasc Imaging. 2017; 18(3):254-227.

16. Monin JL, Quere JP. Low gradient aortic stenosis: Operative risk stratification and predictors for long term outcome. A multicenter study using dobutamine stress hemodynamics. Circulation. 2003;108(3): 319-324.

17. Clavel MA, Dumesnil JG. Outcome of patients with aortic stenosis, small valve area and low flow low gradient despite preserved left ventricular ejection fraction. J Am Coll Cardiol. 2012;60(14):1259-1267.

18. Clavel MA, Messika-Zeitoun D. The complex nature of discordant severe calcified aortic valve disease grading: New insights from combined Doppler echocardiographic and computed tomographic study. J Am Coll Cardiol. 2013;62(24):2329-2338.

19. Takeda S, Rimington $\mathrm{H}$, Smeeton N, Chambers J. Long axis excursion in aortic stenosis. Heart. 2001;86(1):52-56.
20. Hachicha Z, Dumesnil JG, Bogaty P, Pibarot P. Paradoxical low flow, low gradient severe aortic stenosis despite preserved ejection fraction is associated with higher afterload and reduced survival. Circulation. 2007;115(22):2856-2864.

21. Lancellotti P, Donal E, Magne J, et al. Risk stratification in asymptomatic moderate to severe aortic stenosis: The importance of the valvular, arterial and ventricular interplay. Heart. 2010;96(17):1364-1371.

22. Galema TW, Yap SC, Geleijnse ML, et al. Early detection of left ventricular dysfunction by Doppler tissue imaging and N-terminal pro B-type natriuretic peptide inpatients with symptomatic severe aortic stenosis. J Am Soc Echocardiogr. 2008;21(3):257-261.

23. Steine K, Rosseb $\varnothing A B$, Stugaard M, Pedersen TR. Left ventricular systolic and diastolic function in asymptomatic patients with moderate aortic stenosis. Am J Cardiol. 2008;102(7):897-890.

24. Stewart RA, Kerr AJ, Whalley GA, et al; New Zealand Heart Valve Study Investigators. Left ventricular systolic and diastolic function assessed by tissue Doppler imaging and outcome in asymptomatic aortic stenosis. Eur Heart J. 2010;31(18):2216-2222.

25. Geyer H, Caracciolo G, Abe H, et al. Assessment of myocardial mechanics using speckle tracking echocardiography: Fundamentals and clinical applications. J Am Soc Echocardiogr. 2010;23(4):351-369.

26. Lafitte $S$, Perlant M, Reant $P$, et al. Impact of impaired myocardial deformations on exercise tolerance and prognosis in patients with asymptomatic aortic stenosis. Eur JEchocardiogr. 2009;10(3):414-419.

27. Cramariuc D, Gerdts E, Davidsen ES, Segadal L, Matre K. Myocardial deformation in aortic valve stenosis: Relation to the left ventricular geometry. Heart. 2010;96(2):106-112.

28. $\mathrm{Ng} \mathrm{ACT}$, Delgado V, Bertini $\mathrm{M}$, et al. Alterations in multidirectional myocardial functions in patients with aortic stenosis and preserved ejection fraction: A two-dimensional speckle tracking analysis. Eur Heart J. 2011;32(12):1542-1550.

29. Miyazaki S, Daimon M, Miyazaki T, et al. Global longitudinal strain in relation to the severity of aortic stenosis: A two-dimensional speckle tracking study. Echocardiography. 2011;28(7):703-708.

30. Delgado V, Tops LF, van Bommel RJ, et al. Strain analysis in patients with severe aortic stenosis and preserved left ventricular ejection fraction undergoing surgical valve replacement. Eur Heart J. 2009; 30(24):3037-3047.

31. Galli E, Leguerrier A, Flecher E, Leclercq C, Donal E. Increased valvulo-arterial impedance differently impacts left ventricular longitudinal, circumferential and radial function in patients with aortic stenosis: A speckle tracking echocardiography study. Echocardiography. 2017;34(1):37-43.

32. Donal $E$, Bergerot $C$, Thibault $H$, et al. Influence of afterload on left ventricular radial and longitudinal systolic functions: A two-dimensional strain imaging study. Eur J Echocardiogr. 2009;10(8):914-921.

33. Marechaux S, Carpentier E, Six-Carpentier M, et al. Impact of valvuloarterial impedance on left ventricular longitudinal deformation in patients with aortic valve stenosis and preserved ejection fraction. Arch Cardiovasc Dis. 2010;103(4):227-235.

34. Sengupta PP, Taljik AJ. Twist mechanics of the left ventricle: Principles and application. JACC Cardiovasc Imaging. 2008;1(3):366-376.

35. Popescu BA, Calin A. Left ventricular torsional dynamics in aortic stenosis: Relationship between left ventricular untwisting and filing pressures. A two-dimensional speckle tracking study. Eur JEchocardiogr. 2010;11(5):406-413.

36. Voigt JU, Lindenmeier G, Exner B, et al. Incidence and characteristics of segmental post systolic longitudinal shortening in normal, acutely ischemic, and scarred myocardium. J Am Soc Echocardiogr. 2003; 16(5):415-423.

37. Moonen $M$, Lancellotti $P$, Zacharakis $D$, et al. The value of $2 D$ strain imaging during stress testing. Echocardiography. 2009;26(3):307-314.

38. Donal $\mathrm{E}$, Thebault $\mathrm{C}, \mathrm{O}^{\prime}$ Connor K, et al. Impact of aortic stenosis on longitudinal myocardial deformation during exercise. Eur JEchocardiogr. 2011;12(3):235-241.

39. Marechaux S, Ennezat PV, Lelemtel TH, et al. Left ventricular response to exercise in aortic stenosis: An exercise echocardiographic study. Echocardiography. 2007;24(9):955-959.

40. Lindqvist $P, Z$ Zhao Y, Bajraktari G, Holmgren A, Henein MY. Aortic valve replacement normalizes left ventricular twist function. Interact Cardiovasc Thorac Surg. 2011;12(5):701-706.

41. van Dalen BM, Tzikas A, Soliman Ol, et al. Left ventricular twist and untwist in aortic stenosis. Int J Cardiol. 2011;148(3):319-324. 
42. Carasso S, Cohen O, Mutlak D, et al. Relation of myocardial mechanics in severe aortic stenosis to left ventricular ejection fraction and response to aortic valve replacement. Am J Cardiol. 2011;107(7): 1052-1057.

43. Lech AK, Dobrowolski PP, Klisiewicz A, Hoffman P. Exercise-induced changes in left ventricular global longitudinal strain in asymptomatic severe stenosis. Kardiol Pol. 2017;75(2):143-149.

44. Ng ACT, Prihadi EA, Antoni L, et al. Left ventricular global longitudinal strain is predictive off all-cause mortality independent of aortic stenosis severity and ejection fraction. Eur Heart J Cardiovasc Imaging. 2018;19(8):859-867.

45. Kusunose K, Goodman A, Parikh R, et al. Incremental prognostic value of left ventricular global longitudinal strain in patients with aortic stenosis and preserved ejection fraction. Circ Cardiovasc Imaging. 2014;7(6):938-945

46. Lancellotti $P$, Magne J, Donal E, et al. Clinical outcome in asymptomatic severe aortic stenosis: Insights from the new proposed aortic stenosis grading classification. J Am Coll Cardiol. 2012;59(3):235-243.
47. Clavel MA, Webb JG, Rodés-Cabau J, et al. Comparison between transcatheter and surgical prosthetic valve implantation in patients with severe aortic stenosis and reduced left ventricular ejection fraction. Circulation. 2010;122(19):1928-1936.

48. Rost C, Korder S, Wasmeier G, et al. Sequential changes in myocardial function after valve replacement after aortic valve replacement for aortic stenosis by speckle tracking echocardiography. Eur J Echocardiogr. 2010;11(7):584-589.

49. Carasso S, Cohen O, Mutlak D, et al. Differential effects of afterload on left ventricular long and short axis function: Insights from a clinical model of patients with aortic valve stenosis undergoing aortic valve replacement. Am Heart J. 2009;158(4):540-545.

50. Biswas M, Sudhakar S, Nanda NC, et al. Two- and three-dimensional speckle tracking echocardiography: Clinical applications and future directions. Echocardiography. 2013;30(1):88-105.

51. Dweck MR, Joshi S, Murigu T, et al. Midwall fibrosis is an independent predictor of mortality in patients with aortic stenosis. J Am Coll Cardiol. 2011;58(12):1271-1279.

52. Barone-Rochette G, Pierard S, De Meester de Ravenstein C, et al. Prognostic significance of LGE by CMR in aortic stenosis patients undergoing valve replacement. J Am Coll Cardiol. 2014;64(2):144-154. 